\title{
Effects of parafoveal word length and orthographic features on initial fixation landing positions in reading
}

\author{
Patrick Plummer • Keith Rayner
}

Published online: 3 March 2012

(C) Psychonomic Society, Inc. 2012

\begin{abstract}
Previous research has demonstrated that readers use word length and word boundary information in targeting saccades into upcoming words while reading. Previous studies have also revealed that the initial landing positions for fixations on words are affected by parafoveal processing. In the present study, we examined the effects of word length and orthographic legality on targeting saccades into parafoveal words. Long (8-9 letters) and short (4-5 letters) target words, which were matched on lexical frequency and initial letter trigram, were paired and embedded into identical sentence frames. The gaze-contingent boundary paradigm (Rayner, 1975) was used to manipulate the parafoveal information available to the reader before direct fixation on the target word. The parafoveal preview was either identical to the target word or was a visually similar nonword. The nonword previews contained orthographically legal or orthographically illegal initial letters. The results showed that orthographic preprocessing of the word to the right of fixation affected eye movement targeting, regardless of word length. Additionally, the lexical status of an upcoming saccade target in the parafovea generally did not influence preprocessing.
\end{abstract}

Keywords Eye movements · Reading · Orthography · Word perception

For skilled readers, the act of extracting meaning from text seems simple, if not completely effortless. However, this impression belies the complexity of the necessary coordination of perception, attention, motor control, and language

\footnotetext{
P. Plummer $(\bowtie) \cdot K$. Rayner

University of California, San Diego,

La Jolla, CA, USA

e-mail: pplummer@ucsd.edu
}

processing. A comprehensive understanding of eye movement behavior during reading has the potential to elucidate broad aspects of human cognition. Determining what factors influence when and where the eyes move is crucial for understanding written language processing. Despite over a century of research and several decades of investigation using extremely sophisticated instruments and measurements, there is still some contention about what influences the most basic temporal and spatial characteristics of eye movement behavior during reading. In the present study, we further investigated the factors that influence where the eyes go once the decision to move is made. Research on the spatial characteristics of eye movement behavior has the potential to further illuminate the nature of the eye movement targeting system and the constraints imposed on this system during normal reading.

Readers acquire information during fixations, or periods when the eyes are relatively still (Rayner, 1998, 2009). It is important to note that high-spatial-frequency information, such as letters and words, is easily identified in the foveal region. In the region beyond the fovea, the parafovea, because of steep degradation of visual acuity, word and letter information can be extracted, but with greatly reduced efficiency (Schotter, Angele, \& Rayner, 2012). Outside the parafovea, in the peripheral region of the visual field, only low-spatial-frequency information such as word boundary and length information can be extracted. This limits the letter or word identification span (Rayner, Well, Pollatsek, \& Bertera, 1982; Underwood \& McConkie, 1985) for skilled readers to a maximum of about eight letter spaces to the right of fixation. Due to the constraints imposed by visual-acuity limitations, the oculomotor system must plan and execute ballistic eye movements, or saccades, directed to fairly precise locations in text in order to obtain new information or to resample old information. Bringing 
regions of text into the foveal region of the visual field allows readers to reliably identify letters and words. Information specific to local areas of text is extracted from the extrafoveal regions and is utilized during eye movement targeting processes. The mechanisms responsible for targeting the location of the next fixation in text have been found to show sensitivity to various kinds of information.

The spaces between words provide visual cues as to word boundaries and word length that readers are able to extract from parafoveal and peripheral vision. Previous research has demonstrated that word length and word boundary information is used by the eye movement targeting system during reading (Pollatsek \& Rayner, 1982; Rayner, Fischer, \& Pollatsek, 1998). Furthermore, studies have demonstrated that presenting inaccurate word length information or removing spaces between words in the parafovea leads to disruptions in normal reading behavior (Inhoff, Radach, Eiter, \& Juhasz, 2003; Inhoff, Starr, Liu, \& Wang, 1998; Juhasz, White, Liversedge, \& Rayner, 2008; Morris, Rayner, \& Pollatsek, 1990; Rayner et al., 1998; Spragins, Lefton, \& Fisher, 1976). Prior research has also demonstrated that word length positively correlates with the number of fixations received during normal reading, leading to more fixations on long than on short words (Rayner \& McConkie, 1976; Rayner, Sereno, \& Raney, 1996). Previous studies have also well established that distributions of initial fixation locations reflect what is known as the preferred viewing location (PVL), which is located slightly to the left of center for words across all lengths (Rayner, 1979). In a study of word length effects, McDonald (2006) manipulated the visual angles subtended by target words of different lengths in an effort to assess the influences of number of letters and of spatial extent independently. The basic finding was that word-processing differences as a function of length were primarily driven by the number of letters, as opposed to the spatial area of the word. There was also no effect of this spatial manipulation on initial fixation landing position, further demonstrating that word boundary information drives the PVL. However, readers do not always fixate a word at its PVL, because of random motor error and the saccadic range effect (McConkie, Kerr, Reddix, $\&$ Zola, 1988). The saccadic range effect during normal reading captures the tendency for close saccade targets to be overshot and for distant saccade targets to be undershot. There is also abundant evidence that readers extract useful information from words to the right of the word being fixated. The availability of word information in the parafovea has been shown to facilitate the processing of a word once it is fixated; furthermore, denial of accurate information in the parafovea has been shown to significantly disrupt processing. This is conventionally known as the preview benefit (Rayner \& Pollatsek, 1981, 1989; see also Schotter et al., 2012, for a review). Accurate parafoveal previews have also been shown to increase the probability that words will be skipped during first-pass reading (Angele \& Rayner, 2011; Angele, Slattery, Yang, Kliegl, \& Rayner, 2008). In addition, previous research has revealed the particularly important role of initial letter sequences for parafoveal processing (Johnson, Perea, \& Rayner, 2007; Rayner, 1975; White, Johnson, Liversedge, \& Rayner, 2008). Because of the sharp decline in acuity outside the fovea, parafoveal processing is most likely to be affected by the initial letters of parafoveal words. Taken together, these findings raise the question as to what features of a word in the parafovea, aside from its length, might be utilized by the oculomotor system's targeting mechanisms. In particular, what is the role of linguistic (lexical and sublexical) processing on eye movement targeting during normal reading?

Interestingly, many studies have in fact observed small but reliable deviations from the PVL that cannot be accounted for by systematic or random error. Some studies examining the distribution of informativeness within words have suggested that readers target saccades to the most informative regions in a word-specifically, to regions that allow readers to constrain lexical candidates and to distinguish one word from another (Everatt \& Underwood, 1992; Hyönä, Niemi, \& Underwood, 1989; Underwood, Clews, \& Everatt, 1990). The crucial finding in these studies was that initial fixation landing positions were farther into target words when the latter half of the word was more informative than the first half. However, Rayner and Morris (1992) failed to replicate these findings and raised important theoretical and methodological issues with these studies, particularly with regard to morphological processing prior to an initial fixation (see also Hyönä, 1995). Radach, Inhoff, and Heller (2004) also found no effect of morphological complexity on initial fixation landing positions. However, in a study of morphologically complex words, Hyönä and Pollatsek (1998) found that landing positions were closer to word beginnings for noun-noun compound words when the first constituent had low frequency.

Several lines of research have yielded effects on landing positions by manipulating the correctness of target word spellings, orthographic regularity, and orthographic familiarity (Hyönä, 1995; Radach et al., 2004; White \& Liversedge, 2004, 2006a, 2006b). In all of these studies, initial fixation landing positions were found to be closer to word beginnings when the initial letters were difficult to process than when orthographic processing was relatively easy. Other studies have revealed no effect on landing positions when manipulating foveal processing load (White \& Liversedge, 2006a), target word contextual predictability (Rayner, Binder, Ashby, \& Pollatsek, 2001), or manipulations of length and predictability (Rayner, Slattery, Drieghe, \& Liversedge, 2011). It is important to note that nearly all of the critical words in these studies were relatively long, raising the question of whether word length would modulate any of the observed landing position effects. 
Using short target words embedded in neutral sentences, White (2008) failed to observe any effects of orthographic familiarity or lexical frequency on initial fixation locations. To reconcile those results with previous work that had observed orthography affecting landing positions, she suggested that the influence of orthographic characteristics on saccade targeting might differ across long and short words. Because all of her critical words were relatively short (four or five letters), this possibility could not be explored using her data. One might expect word length to influence the effect of initial letter information on saccade targeting for a variety of reasons. Since empirical evidence has indicated that both random and systematic oculomotor error probably have proportionally larger impacts on the processing of short than of long words, it seems plausible that a sensitive and flexible targeting system might compensate for this by employing different strategies for programming saccades into words of different lengths. This is not to say that letter information should be extracted differently as a function of word length, only that letter information might exert a different influence (i.e., be utilized more or less) as a function of word length. For instance, the eye movement targeting system may not use available letter information when accounting for the increased likelihood that all of a relatively short word's letters will be foveated once the eyes saccade into the word. This might explain why informativeness effects have only been observed for long words. One would not expect to see informativeness affect landing positions in short words, since all of the letters will most likely fall in a region of high visual acuity once the word is fixated. This would eliminate the need to strategically target saccades to portions of a letter string that would ordinarily fall outside the average letter identification span in an effort to maximize word identification speed. If targeting a region of potential orthographic processing difficulty would lead to a less-than-optimal viewing position for the entire word, the eye movement control system might forgo the use of orthographic information for saccade-targeting processes. This would more likely be the case for long words, since the site of orthographic processing difficulty could then be relatively far from the PVL. The saccade-targeting system may also be sensitive to the increased likelihood that a relatively long word will ultimately receive more than one first-pass fixation. This might result in the programming of saccades directly to the site of orthographic difficulty, irrespective of its distance from the PVL.

In sum, prior research has yielded mixed results with regard to the roles of lexical and sublexical processing on saccade characteristics. Previous studies have defined, operationalized, and manipulated orthographic familiarity, informativeness, legality, saliency, and regularity in a variety of ways in an attempt to highlight the possible role of orthographic characteristics as important driving factors behind the observed differences in initial fixation landing positions (Everatt \& Underwood, 1992; Hyönä et al., 1989; Inhoff et al., 2003; Radach et al., 2004; Underwood et al., 1990; White, 2008; White \& Liversedge 2004, 2006b). Across many experiments, areas of text containing difficult-toprocess or visually "salient" low-level linguistic information seem to be strongly correlated with deviations from predicted spatial saccade characteristics (i.e., deviation from the PVL). Only a few theoretical accounts have addressed how parafoveal word characteristics might result in initial landing positions closer to the beginning of the word. One possible source of landing position effects could be linguistic difficulty at the word or discourse level. It has been posited that any processing difficulty induced by word or text characteristics may influence spatial eye movement control (Hyönä, 1995; Hyönä \& Pollatsek 1998, 2000). By this account, word length should influence landing positions, since other lexical characteristics that modulate processing difficulty tend to differ as a function of word length. A word's orthographic neighborhood size, for example, reflects the number of words that contain similar letter sequences and is negatively correlated with word length. Previous reading studies have observed effects whereby higher-frequency orthographic neighbors, as well as relatively large neighborhood sizes, elicit processing disruption for target words (Perea \& Pollatsek, 1998; Pollatsek, Perea, \& Binder, 1999). As a result of increased lexical competition and activation of associated orthographic representations, large orthographic neighborhoods for a word could disrupt orthographic processing and mediate the influence of orthographic characteristics on saccade targeting. Lexical frequency is another well-known and important lexical variable. Generally, long words have lower lexical frequencies and take longer to process than do short words. To the extent that lexical processing difficulty affects saccade targeting, long and short words should tend to differ in the relative positions of initial fixations, even when standardizing across different character lengths.

Another account of observed landing position effects assumes that words containing initial letter sequences "pop out" due to irregularity or unfamiliarity (Beauvillain, Doré, \& Baudouin, 1996; Findlay \& Walker, 1999; Hyönä, 1995). This account might also predict interactive word length and letter influences on landing positions. The initial letters, which are often extracted during parafoveal preview, constitute a larger proportion of short than of long words. Thus, orthographic irregularities could be more likely to attract the attention of the word-processing and saccade-targeting systems in relatively short words. In all, several factors at different levels of language processing may affect saccade targeting and produce interactive effects of word length and orthographic information. 
Prior research has been informative as to the influence of linguistic processing on where the eyes move. Still, there is no well-developed theoretical account for why these small but seemingly reliable effects on landing positions seem to be inseparably linked to (but not necessarily limited to) orthographic characteristics. It is also true that none of the theoretical accounts of effects on saccade targeting have spoken directly to the possible influences of word length. Since parafoveal word length processing clearly plays a role in saccade targeting, and since linguistic information is extracted during the time when saccades are targeted, further examination of parafoveal linguistic processing may help to engage this empirical trend. No previous study has utilized a controlled manipulation of word length and of the orthographic legality of initial letters. Testing the specific characteristics of orthographic effects in the context of word length could help to make these empirical trends clearer. In an effort to further illuminate the nature of orthographic processing of parafoveal words, we investigated the roles of word length and orthographic features on parafoveal processing and saccade target programming. More specifically, in the present study, we investigated how eye movement behavior is affected by the orthographic legality of initial letter sequences and tested whether word length systematically constrains the extraction and processing of parafoveal letter information. In addition, the study further examined the effects of word length and word boundary information on eye movement behavior.

\section{Present experiment}

In the present experiment, we investigated orthographic legality effects on saccade programming using a design that would be sensitive to any modulatory effects of word length. For the purposes of this study, orthographic legality is operationally defined as the extent to which a sequence of letters conforms to the general rules of the written English language. To achieve the desired experimental design, the gaze-contingent invisible boundary paradigm (Rayner, 1975) was employed to manipulate parafoveal preview. In this paradigm, a particular letter string was displayed in place of the target word until the eyes moved past an invisible boundary, which prompted the replacement of the preview by the target word. An examination of potential word length effects on parafoveal orthographic processing was achieved using frequency-matched long-short word pairs that shared identical initial letter strings. By employing the nonword orthographic legality manipulation, the present study design provides the potential for a detailed analysis of lexical and sublexical parafoveal processing. Using the boundary paradigm, initial letter sequences for target words were altered in such a way as to produce (1) a nonword with illegal orthography or (2) a nonword with legal orthography; in addition, a further condition was based on (3) a parafoveal preview identical to the target word. Because identical previews contain actual words, which necessarily conform to orthographic rules, legal nonword and identical previews have much higher orthographic regularity and pronounceability than do illegal nonword previews. Since orthographic information about upcoming words is most likely to be extracted from initial letters, manipulation of the beginning of words should yield the strongest experimental effect. Given that word length information can be easily extracted from nonfoveal regions, effects associated with parafoveal word length will have the potential to interact with parafoveal orthographic processing. Critically, the use of the boundary paradigm enabled us to distinguish parafoveal and foveal processing and to detect any systematic changes in saccade target processing as a function of orthographic legality and length.

If parafoveal processing of orthographic legality affects the targeting of landing positions for saccades into upcoming words, the parafoveal manipulation should result in different patterns of data across preview conditions. Specifically, landing positions for illegal nonword previews should significantly deviate from the PVL when compared to those for identical and legal nonword previews. This would provide strong evidence that conventional saccade programs, aiming fixations at the centers of words, can be adjusted to target sites of anomaly or of potential orthographic processing difficulty. If the lexical status of a preview string, whether word or nonword, affects saccade programming, then the landing positions for both legal and illegal nonwords should differ significantly from those for full previews. If orthographic legality and lexical status do not have reliable effects on spatial eye movement control, no differences should be detected across preview conditions. If word length systematically constrains saccadetargeting processes or the extraction of parafoveal information, different patterns of results should be observed across the length conditions.

\section{Method}

\section{Subjects}

A group of 36 undergraduates at the University of California, San Diego, participated for course credit. All subjects were native English speakers with normal or corrected-to-normal vision who were naïve about the research goals.

\section{Stimuli}

The experimental stimuli consisted of 60 frequencymatched long-short word pairs with identical initial trigram letter sequences (e.g., actor-activist). Lexical frequencies 
for all stimuli (target and pretarget words) were computed via log-transformed HAL frequency norms (Lund \& Burgess, 1996) using the English Lexicon Project (Balota et al., 2007). The range of target word frequencies was 5-11, with an average frequency of 7.91. Long target words contained either eight or nine letters $(M=8.3)$ and had an average $\log$ frequency of 7.83 (4,938 counts per million). Short target words contained either four or five letters $(M=$ 4.72) and had an average log frequency of 7.99 (5,748 counts per million). The differences in lexical frequency for each long-short pair were always less than one $\log$ frequency count. In an attempt to avoid effects associated with morphological complexity, no target nouns or pretarget adjectives were compound words. Nevertheless, there were morphologically complex words in both the long and short target word conditions. The average number of morphemes comprising the short target words was $1.39(S D=0.49)$ morphological units, and the long target words averaged $2.18(S D=0.75)$ morphological units. Standard $t$ tests revealed significant differences in the average number of morphological units $[t(118)=6.904, p<.01]$.

The parafoveal preview available to the reader either was identical to the target word (full) or was a visually similar nonword. The nonword previews contained either orthographically legal letter sequences (legal) or orthographically illegal and unpronounceable (illegal) initial letter sequences created by substituting the second and third letters of the target word. The letter substitutions used to create the nonword parafoveal previews were identical for the long and short targets of each item. In this design, orthographic legality was necessarily confounded with familiarity and regularity, in that orthographic structures that conform to the rules of English language will have an inherently higher frequency than will illegal and irregular orthographic structures. To assess the orthographic regularity and familiarity of the parafoveal preview strings, $n$-gram frequencies, as well as orthographic neighborhood sizes, were calculated using the CELEX English Lexical Database (Baayen, Piepenbrock, \& Gulikers, 1995) via N-Watch (Davis, 2005). In addition, Bigram Frequency $\times$ Position values were calculated using the English Lexicon Project (Balota et al., 2007). For this study, $n$-grams represented letter sequences, such that hats has three bigrams - ha, at, and ts - and two trigrams - hat and ats.

Bigram token frequency is computed as the sum of the frequencies for lexical items that contain a particular twoletter sequence in a specific position. Bigram type frequencies represent the number of lexical items of the same length that contain a particular two-letter sequence in a specific position. Bigram Frequency $\times$ Position represents a sum of bigram frequencies, counting only instances in which the bigram appears in a specific position. Orthographic neighborhood is defined as the number of words that can be obtained by changing one letter while preserving the identities and positions of the other letters. For example, the English Lexicon Project reports such orthographic neighbors for CAT as COT, HAT, and CAM (Balota et al., 2007; see Table 1). The critical experimental manipulation was performed on the initial trigram of each target word. Table 2 displays the average Bigram Frequency $\times$ Position for the initial trigrams of target word previews. These averages were the same across length conditions, since the long-short

Table 1 Preview letter string by length condition: Average sublexical frequency means and orthographic neighborhoods for entire preview letter strings, grouped by length condition

\begin{tabular}{|c|c|c|c|c|c|c|}
\hline & \multicolumn{3}{|l|}{ Short } & \multicolumn{3}{|l|}{ Long } \\
\hline & Full & Legal & Illegal & Full & Legal & Illegal \\
\hline BG_TK & $1,157(1,116)$ & $863(890)$ & $350(512)$ & $532(255)$ & 464 (264) & $391(255)$ \\
\hline BG_TP & $30(37)$ & $22(16)$ & $11(14)$ & $75(35)$ & $66(35)$ & $56(35)$ \\
\hline TG_TK & $206(120)$ & $48(151)$ & $8(30)$ & 139 (133) & $116(130)$ & 108 (127) \\
\hline TG_TP & $4.7(2.9)$ & $1.7(2.4)$ & $0.4(1.4)$ & $17.8(13.1)$ & $14.8(12.5)$ & $13.9(12.4)$ \\
\hline BG_F_P & $1,315(498)$ & $906(531)$ & $373(348)$ & $2,460(760)$ & $2,024(749)$ & $1,461(596)$ \\
\hline Ortho_N & $4.33(4.78)$ & $2.23(3.04)$ & $0.22(0.52)$ & $0.2(0.48)$ & $0.03(0.18)$ & $0.0(0.0)$ \\
\hline
\end{tabular}

Standard deviations are provided in parentheses. Within length conditions, $t$ tests were performed across preview conditions for each measure. All bigram and trigram averages were significantly different across short target word previews [all $t \mathrm{~s}(118)>2.01$, all $p \mathrm{~s}<.05$ ], except for full as compared to legal previews in bigram token and type frequencies [both $t \mathrm{~s}(118)<1.60$, both $p \mathrm{~s}>.10$ ]. In the long target word conditions, only full and illegal previews differed significantly for bigram token and type frequencies [both $t \mathrm{~s}(118)>2.97$, both $p \mathrm{~s}<.01$ ], all other comparisons yielded no significant differences [all $t \mathrm{~s}(118)<1.70$, all $p \mathrm{~s}>.09$ ]. The lack of significant differences across preview conditions is attributable to the influence of the unaltered bigrams after the initial trigram. For orthographic neighborhood size, all averages across previews for short targets differed significantly [all $t \mathrm{~s}(118)>2.86$, all $\mathrm{ps}<.05$ ]. For long target previews, the full versus legal and full versus illegal conditions differed significantly [both $t \mathrm{~s}(118)>2.55$, both $p \mathrm{~s}<.05]$, but legal versus illegal previews did not $[t(118)=1.29, p=.20]$. BG_TK, bigram token frequency; BG_TP, bigram type frequency; TG_TK, trigram token frequency; TG_TP, trigram type frequency; BG_F_P, Bigram Frequency $\times$ Position; Ortho_N, orthographic neighborhood size 
Table 2 Initial trigram of preview: Average Bigram Frequency $x$ Position measures for the initial trigram of the preview for both long and short target words

\begin{tabular}{llll}
\hline & Full & Legal & Illegal \\
\hline Bigram Frequency $\times$ Position & $4.63(3.19)$ & $3.45(2.27)$ & $0.11(0.26)$ \\
\hline
\end{tabular}

Standard deviations are provided in parentheses. Paired comparisons revealed significant differences across preview conditions [all $t \mathrm{~s}(118)>$ 5.38$, all $p \mathrm{~s}<.01]$

target word pairs were matched for initial trigram identity and since identical manipulations were performed for each member of the target word pair.

\section{Design}

Either the long or the short item from the target word pair was embedded in a neutral sentence frame (see Fig. 1). In all of the sentence frames, target words were always preceded by a six- to nine-letter adjective $(M=7.65)$ with log frequency ranging from 6 to $12(M=9.04)$. Target words in all sentence frames were followed by a word at least four letters long, in order to capture any potential spillover effects. To ensure a lack of differences across the length conditions, 11 undergraduates from the University of California, San Diego, who were not subjects in the eyetracking study, performed a standard cloze task for each sentence frame after being presented with the sequence of words up to the target word's position. Short target words were correctly predicted an average of $0.3 \%$ across items $(S D=1.6 \%)$. Long target words were correctly predicted an average of $1.1 \%$ across items $(S D=5.3 \%)$. There were no significant differences in the average predictability of the long and short target words given the prior sentence frames $[t(118)=1.054, p=.29]$. An additional 20 undergraduates from the University of California, San Diego, who were also not subjects in the eyetracking study, rated the goodness of fit for either the long or the short target word in the context of the prior sentence frame for each item. Subjects were asked to rate how well the target word fit grammatically and plausibly with the preceding word sequence, on a scale of $1-7$, with 1 being very poor and 7 being very good. The goodness-of-fit ratings averaged $5.48(S D=1.89)$ for short target words and $5.49(S D=1.88)$ for long target words; again, there was no significant difference in the average goodness of the target words fits across the length conditions $[t(1198)=0.123, p=$ .90]. Counterbalanced assignments of the sentence frames to the length and preview $(2 \times 3)$ conditions were accomplished using a Latin square design, resulting in six lists of the 60 sentence frames. Each subject was exposed to each experimental condition an equal number of times; however, no subject read any sentence frame more than once. The experimental sentences were presented with 40 filler sentences in a randomized order, and all sentences were preceded by five practice trials. Each sentence was followed by a true-false comprehension question.

\section{Procedure}

Eye movements were recorded from the right eye of each subject using a tower-mounted SR Eyelink 1000 eyetracking system with a sampling rate of $1000 \mathrm{~Hz}$. The stimuli were presented on a 20-in. NEC MultiSync FP1370 CRT Desktop Monitor at a distance of $55 \mathrm{~cm}$, with a display resolution of $1,024 \times 768$ and a refresh rate of $150 \mathrm{~Hz}$. At the beginning of each session, the tracker was calibrated using a nine-point procedure; the calibration was checked after each trial, and recalibration was performed when necessary. Subjects were seated in a well-lit room with their head movements minimized by a chinrest and forehead rest. They were instructed to read the sentences silently at a normal and comfortable pace and to answer the subsequent true-false questions using a

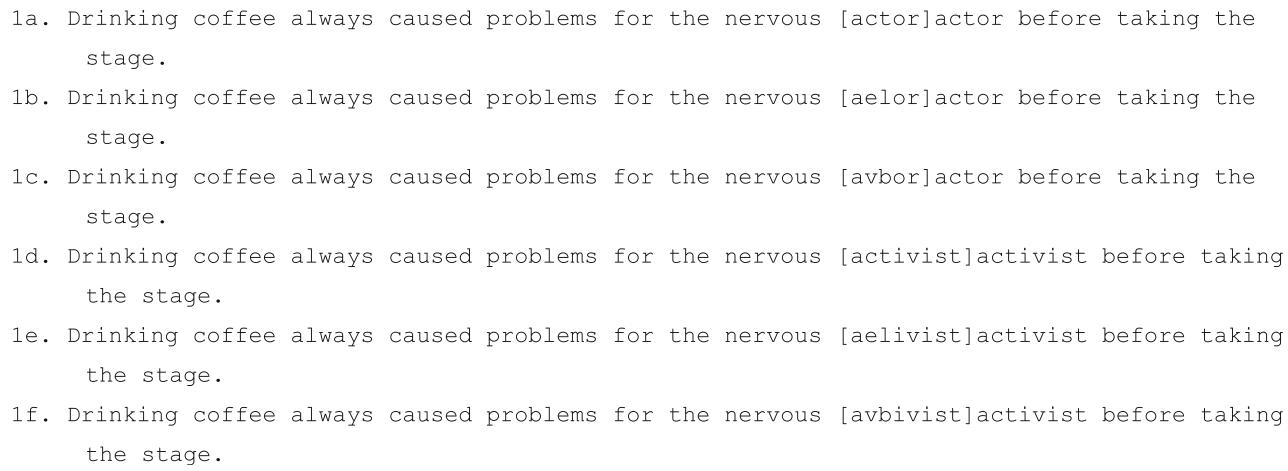

Fig. 1 Examples of a long-short target pair under all experimental conditions. The letter string in brackets in each sentence provides the parafoveal preview that is displayed before the reader crosses the invisible boundary. Upon boundary crossing, the correct target word is presented. Sentences $1 \mathrm{a}$ and $1 \mathrm{~d}$ are examples of the full-preview conditions; $1 \mathrm{~b}$ and $1 \mathrm{e}$ are examples of the orthographically legal nonword preview conditions; and $1 \mathrm{c}$ and $1 \mathrm{f}$ are examples of the orthographically illegal nonword preview conditions 
handheld response pad. Subject accuracy on comprehension questions was very high $(M=90 \%, S D=6 \%)$. The experimenter monitored eye movements throughout the session. The sentences were presented from the left-center of the display screen after subjects had fixated a drift correction point in that position. All sentences and questions were presented using monospaced Courier New 14-pt. font with 1 deg of visual angle subtending 2.2 characters and one character being 11 pixels wide. The invisible boundary was placed at the beginning of the space immediately to the left of the target word. All target words were presented close to the center of the screen. Display changes occurred an average of $9 \mathrm{~ms}(S D=2 \mathrm{~ms})$ after the boundary crossing had been detected. All trials on which the subject blinked during first-pass reading of the critical region - consisting of the pretarget adjective, the target word, and the word after the target-were excluded from the analysis. In addition, all trials on which the display change occurred prior to the first saccade that crossed the invisible boundary or after the onset of the first fixation to the right of the invisible boundary were excluded from the analysis. By these criteria, $11 \%$ of the data were lost ( 230 of 2,160 trials). Seven subjects (19\%) reported awareness of at least one display change. Each subject was exposed to exactly 60 display changes, 40 of which were visible; however, no subjects reported awareness of more than four display change events.

\section{Analysis of eye movements}

Reading time measures were calculated after eliminating outliers with durations less than $40 \mathrm{~ms}$ or greater than $900 \mathrm{~ms}$ (two trials were lost due to the upper, and one to the lower, limit). The measures of interest followed generally accepted definitions (Rayner, 1998). First-pass fixations are fixations after the eyes first enter a word until they move off the word, given that they had not progressed beyond that word before the first fixation. Single-fixation duration (SFD) is the duration of the initial first-pass fixation on a word when the word has received only one firstpass fixation. First-fixation duration (FFD) is the duration of the initial first-pass fixation on a word, regardless of whether there have been subsequent first-pass fixations on the word. Gaze duration (GD) is the sum of all first-pass fixation durations on a word before leaving that word to the right or the left. The go-past time (also called the regression path duration) for a word is the sum of all fixation durations from the initial first-pass fixation on that word until the eyes have moved beyond the word's right boundary, including all regressive fixations on words to the left. The number of firstpass fixations (NFix) is the total number of fixations a word receives after first being encountered until the word region is exited to the left or the right. This particular measure also captures the probability of refixation during first-pass reading. The first-pass skipping rate (Skip) for the target word is calculated as the proportion of trials on which the target word was not fixated at all or was only fixated after a subsequent word had been fixated. A separate measure of first-pass skipping proportion (Restricted Skip) was calculated after reclassifying first-pass skips that were immediately followed by a fixation on the target word. This particular behavior is conventionally thought to reflect oculomotor error in saccade targeting (Drieghe, Rayner, \& Pollatsek, 2005). An analysis of variance (ANOVA) was performed for each of the dependent measures. Effects were analyzed using subjects $\left(F_{1}\right)$ and items $\left(F_{2}\right)$ as random factors.

\section{Analysis of landing position}

Using the previously stated selection criteria, landing positions on the target words were calculated as proportions through the word region of the initial fixation. Reporting landing position in terms of the proportion through the word, as opposed to the number of characters into the word, allowed for a more meaningful comparison of the results across the two length conditions. Landing position was measured from the first pixel of the first character in the target word and included five pixels of blank space after the last pixel of the last character in the word. For completeness, the landing position results will also be reported in character spaces into the target. Saccade length is the amplitude, measured in character spaces, for the saccade first entering the target region. Launch site, measured in character spaces, is the total number of characters away from the target word's left boundary (as defined for the landing position analysis) for the starting point of the saccade first entering the target word region. Conventional ANOVAs across subjects $\left(F_{1}\right)$ and items $\left(F_{2}\right)$ were conducted for ease of comparison to the results of previous landing position studies. In addition, linear mixed-effects models were used to analyze the landing position results.

\section{Results}

Landing position

Table 3 displays landing position averages for first fixations across conditions. A main effect of target word length was observed $\left[F_{1}(1,35)=45.99, p<.01 ; F_{2}(1,59)=\right.$ $30.69, p<.01]$. The proportions through the target averaged .465 for short words and .397 for long words; in terms of character spaces, these were equivalent to 2.33 characters for the short words and 3.44 characters for the long words. Preview condition for the target word also showed a main effect on the initial fixation landing position $\left[F_{1}(2,70)=5.02, p<.01 ; F_{2}(2\right.$, $118)=4.62, p<.01]$. The average proportions through the target word of the initial landing position were .448 for full previews, .424 for orthographically legal previews, and .407 for 
Table 3 Critical saccade characteristics

\begin{tabular}{|c|c|c|c|c|c|c|}
\hline & \multicolumn{3}{|l|}{ Short } & \multicolumn{3}{|l|}{ Long } \\
\hline & Full & Legal & Illegal & Full & Legal & Illegal \\
\hline LP prop & $.489(.263)$ & $.451(.245)$ & $.430(.264)$ & $.406(.202)$ & $.398(.210)$ & $.383(.210)$ \\
\hline LP char & $2.5(1.5)$ & $2.3(1.4)$ & $2.2(1.5)$ & $3.5(1.8)$ & $3.5(1.9)$ & $3.3(1.9)$ \\
\hline Launch site & $5.5(3.0)$ & $5.8(3.0)$ & $6.0(3.3)$ & $5.9(3.5)$ & $6.0(3.3)$ & $5.9(2.9)$ \\
\hline Saccade length & $8.1(2.9)$ & $8.1(2.8)$ & $8.2(2.9)$ & $9.5(3.2)$ & $9.5(2.9)$ & $9.2(2.8)$ \\
\hline
\end{tabular}

First-fixation landing positions are reported in terms of the average proportion through the target, beginning from its left boundary (LP prop). Landing positions are also reported in terms of average character spaces into the target, beginning from its left boundary (LP char). The launch site measure presents the average distance from a target word's left boundary of the fixation prior to the saccade entering the target word. The saccade length measure is presented as the average amplitude in character spaces. Standard deviations are provided in parentheses

orthographically illegal previews; in terms of character spaces, the character positions for first fixations on target averaged 3.03 characters for full previews, 2.92 for orthographically legal nonwords, and 2.78 for orthographically illegal nonwords. Using Tukey's HSD test to detect the specific nature of the preview effect revealed that landing positions for the full previews were significantly different from landing positions for the orthographically illegal previews across both subjects $(p<.01)$ and items $(p<.01)$. Landing positions on orthographically legal previews did not differ significantly from full or orthographically illegal previews across subjects or items (Tukey's HSD, all $p s>$.15). Importantly, no interactions of word length and parafoveal preview were observed for landing positions across subjects or items (all $F_{\mathrm{s}}<1$ ). These results suggest that orthographic features influence saccade targeting in a graded, stepwise fashion and that there were no systematic differences in orthographic processing across saccade targets of different lengths. While there were significant preview condition effects, these suggest that lexical status and orthographic legality affect saccade characteristics independently. Orthographic regularity, which is closely tied to both legality and lexical status, provides a more suitable dimension on which to assess the landing position effects.

A linear mixed-effects regression was conducted that included by-subjects and by-items random intercepts, Subject $\times$ Experimental Condition random slopes, and a full factorial analysis of length and preview. In addition, the model included saccade launch site as a continuous predictor of initial landing position. Including the last parameter allowed us to account for the influence of saccade launch site when assessing the effects of preview and length conditions. All parameters were set using restricted maximum likelihood (REML) estimations. In this more comprehensive landing position analysis, target word length yielded a significant effect on landing position $[F(1,31)=30.66, p<.01]$. Preview condition of the target word also maintained a significant effect on initial fixation landing positions $[F(2,67)=5.79, p<.01]$. As before, there was no significant interaction of length and preview $[F(2$, $1643)=0.72]$. The launch site of the critical saccade into the target word had a significant effect on initial fixation location $[F(1,1769)=371.56, p<.01]$. These results indicate that orthographic preprocessing influences eye movement targeting mechanisms during normal reading. Furthermore, this influence is not systematically constrained by word length. Crucially, the pattern of significant effects remained even when accounting for the influence of saccade launch site. ${ }^{1}$

Launch site and saccade length

The spatial characteristics of eye movements leading to the initial fixation of the target word were analyzed. Results for all first fixations on the target word are presented below and in Table 3. The launch site of the first saccade entering the target word region was calculated as the number of characters to the left of the leftward boundary of the target. The subject and item analyses failed to show significant main effects for length or preview condition on critical saccade launch site (all $F_{\mathrm{S}}<1$ ). There were was also no interaction of length and preview for launch site into the target region (all $F \mathrm{~s}<1.6)$. The amplitude of the saccade entering the target word was calculated as the distance, in letter spaces, between the location of the first fixation on the target word and the immediately preceding fixation. Saccade length yielded a significant main effect of target word length: Saccades into long target words averaged 9.4 characters in length, whereas saccades into short target words averaged 8.1 characters $\left[F_{1}(1,35)=88.96, p<.01 ; F_{2}(1,59)=87.75\right.$, $p<.01]$. Although the average saccade amplitude and critical saccade launch site did differ across preview conditions (see Table 3), due to high variability there were no significant effects. No main effect of preview condition or

\footnotetext{
${ }^{1}$ An additional analysis of landing positions restricted to only trials on which the target word received exactly one fixation was conducted using both a standard ANOVA and a linear mixed-effects modeling approach identical to the unrestricted first-fixation landing position analysis. The patterns of significant results were identical to those found in the unrestricted landing position analysis for both statistical approaches.
} 
Table 4 Average single-fixation durations on the adjective preceding the target word

\begin{tabular}{lll}
\hline & Short & Long \\
\hline Full & $235(77)$ & $237(84)$ \\
Legal & $227(73)$ & $232(81)$ \\
Illegal & $224(77)$ & $223(91)$ \\
\hline
\end{tabular}

Standard deviations are provided in parentheses

interactions of length and preview were found to be significant across subjects or items [all $F_{\mathrm{s}}<1.22$ ].

Reading times on the word before the target

Single-fixation durations on the pretarget adjective (see Table 4) showed a main effect of preview condition, whereby durations on the adjectives differed significantly as a function of preview condition. Average durations on the adjectives preceding targets with identical previews $(238 \mathrm{~ms})$ were longer than those with legal previews $(231 \mathrm{~ms})$, and durations on adjectives preceding both legal nonword and identical previews were longer than those before illegal previews ( $223 \mathrm{~ms})$ $\left[F_{1}(2,70)=5.11, p<.01 ; F_{2}(2,118)=4.72, p<.01\right]$. No other dependent measures showed effects of length or preview across items or subjects (all $F_{\mathrm{S}}<1$ ). Tukey's HSD demonstrated that average single-fixation durations on the adjective preceding the target for full as compared to illegal nonword previews differed significantly $(p<.05)$. Legal nonword previews differed numerically from illegal nonword and full previews; however, this effect was not significant (both $p$ s $>$ .35). These results show that the orthographic characteristics of an upcoming word have a small but reliable effect on single-fixation durations. Orthographic difficulty in the parafovea decreased fixation durations on pretarget adjectives; however, neither lexical status nor orthographic legality could account for this effect independently. Interestingly, this effect was only observed on the fixation immediately preceding the first fixation on the target when the pretarget adjective received only one first-pass fixation.

First-pass reading measures for the target word

Table 5 displays the average first-pass reading time measures for all conditions. Three first-pass reading times where analyzed: first-fixation duration, single-fixation duration, and gaze duration. Word length had an effect on target word gaze duration, such that shorter target words averaged $292 \mathrm{~ms}$, whereas long target words averaged $316 \mathrm{~ms}$ $\left[F_{1}(1,35)=20.79, p<.01 ; F_{2}(1,59)=14.09, p<.01\right]$. Word length had no significant effect on first-fixation or single-fixation durations (all $F_{\mathbf{S}}<1$ ). All first-pass reading times showed significant effects of preview condition [for FFD, $F_{1}(2,70)=8.27, p<.01 ; F_{2}(2,118)=7.85, p<.01$; for SFD, $F_{1}(2,70)=8.73, p<.01 ; F_{2}(1,118)=8.27, p<$ .01 ; for GD, $F_{1}(2,70)=9.19, p<.01 ; F_{2}(2,118)=8.24, p<$ $.01]$. First-pass reading times, by all measures, were longest in the illegal nonword preview conditions and shortest in the full-preview conditions, with legal nonword preview conditions falling in between. Tukey's HSD revealed that fullpreview conditions yielded significantly shorter first fixations, single fixations, and gaze durations than did orthographically legal nonword preview conditions (all FFD, SFD, and GD analyses: $p<.05$ for both subjects and items). Full-preview conditions also resulted in significantly shorter reading times on target words than did orthographically illegal nonword preview conditions (all FFD, SFD, and GD analyses: $p<.01$ for both subjects and items). No significant

Table 5 Reading measures on the target word

\begin{tabular}{|c|c|c|c|c|c|c|}
\hline & \multicolumn{3}{|l|}{ Short } & \multicolumn{3}{|l|}{ Long } \\
\hline & Full & Legal & Illegal & Full & Legal & Illegal \\
\hline FFD & $249(4.8)$ & $270(5.5)$ & $271(5.3)$ & $249(5.2)$ & $267(4.8)$ & $265(4.7)$ \\
\hline SFD & $256(5.3)$ & $281(6.2)$ & $285(6.2)$ & $266(6.4)$ & $278(5.5)$ & $282(5.8)$ \\
\hline GZD & $282(6.6)$ & $298(6.4)$ & $309(6.4)$ & $303(6.8)$ & $320(6.6)$ & $330(7.7)$ \\
\hline Skip & $.138(.019)$ & $.123(.018)$ & $.120(.018)$ & $.036(.010)$ & $.015(.006)$ & $.009(.004)$ \\
\hline Restricted skip & $.102(.017)$ & $.077(.015)$ & $.071(.014)$ & $.027(.009)$ & $.008(.004)$ & $.005(.003)$ \\
\hline NFix & $1.17(.02)$ & $1.17(.02)$ & $1.22(.03)$ & $1.30(.03)$ & $1.32(.03)$ & $1.35(.03)$ \\
\hline Go-past & 351 (14.7) & $383(15.5)$ & $400(15.2)$ & $370(13.2)$ & $412(16.0)$ & 409 (12.7) \\
\hline TTime & 384 (11.2) & 407 (13.4) & $402(11.2)$ & 423 (13.6) & 457 (14.4) & $496(16.7)$ \\
\hline
\end{tabular}

Reading times on the target word are all presented in milliseconds. Target word skipping and restricted skipping rates are presented as the proportions of trials on which the target word did not receive a first-pass fixation. All measures report standard errors in parentheses. FFD, firstfixation duration; SFD, single-fixation duration; GZD, gaze duration; NFix, average number of first-pass fixations on the target word; TTime, total time of all fixations on target word 
differences were observed between orthographically legal previews and orthographically illegal previews for subjects or items (all $p \mathrm{~s}>.21$ ), and no interactions of length and preview were found for any first-pass reading time measure (all $\left.F_{\mathrm{s}}<1\right)$. These results suggest that readers received a significant preprocessing benefit when the target word was fully available for parafoveal preview, as compared to all nonword previews. Moreover, no preprocessing benefit was observed for nonwords exhibiting orthographic legality rather than illegality. Taken together, these results demonstrate an effect of lexical status but not of orthographic legality in first-pass reading.

The number of first-pass fixations on the target word was also analyzed. Target word length had a significant effect, such that long words received more fixations during first-pass reading $(1.18$ vs. 1.32$)$ than did short words $\left[F_{1}(1,35)=\right.$ $\left.39.90, p<.01 ; F_{2}(1,59)=35.61, p<.01\right]$. Preview condition had no significant effect on the number of first-pass fixations (all $F$ s $<2.3$ ), nor did we find an interaction of length and preview for any of the four first-pass measures (all $F_{\mathrm{S}}<1$ ).

Intermediate and late reading measures

Two measures thought to reflect relatively intermediate or late processing, go-past duration and total reading time, were examined. Preview condition for the target word had a significant effect on go-past reading times $\left[F_{1}(2,70)=\right.$ $\left.5.06, p<.01 ; F_{2}(2,118)=5.32, p<.01\right]$. The average gopast reading times were $358 \mathrm{~ms}$ for full previews, $394 \mathrm{~ms}$ for orthographically legal nonword previews, and $401 \mathrm{~ms}$ for orthographically illegal previews. The effect of target word length resulted in go-past reading times averaging $23 \mathrm{~ms}$ longer for long than for short target words. This effect was significant by subjects $\left[F_{1}(1,35)=3.97, p=.046\right]$ but only marginal by items $\left[F_{2}(1,59)=3.20, p=.074\right]$. We found no statistically significant interactions of word length and preview effects (all $F \mathrm{~s}<1$ ). Total reading times on target words showed a main effect of length for both subjects and items $\left[F_{1}(1,35)=39.62, p<.01 ; F_{2}(1,59)=34.42, p<.01\right]$ : Long words were fixated an average of $63 \mathrm{~ms}$ longer than short words. The nature of the parafoveal preview also had a significant influence on total reading times, which averaged $403 \mathrm{~ms}$ when previews were identical to the target words, $431 \mathrm{~ms}$ when the previews were nonwords with high orthographic legality, and $448 \mathrm{~ms}$ when the previews were orthographically illegal nonwords $\left[F_{1}(1,35)=6.35, p<.01\right.$; $\left.F_{2}(1,59)=6.03, p<.01\right]$. The interaction of word length and parafoveal preview approached marginal significance $\left[F_{1}(2,70)=2.76, p=.064 ; F_{2}(2,118)=2.47, p=.084\right]$. The total reading times for long target words increased numerically across preview conditions, whereas smaller differences in total reading times were observed for short target words across preview conditions (see Table 5).
Target word skipping

As would be expected, target word length yielded a robust effect on first-pass skipping probability $\left[F_{1}(1,35)=102.6\right.$, $\left.p<.01 ; F_{2}(1,59)=89.05, p<.01\right]$ : Short target words were skipped $12 \%$ of the time, whereas long target words were skipped $2 \%$ of the time. Preview condition did not yield significant effects on first-pass skipping rates (all $F_{\mathrm{s}}<1.78$ ). However, after reclassifying skips immediately followed by regressions into the target word, both length $\left[F_{1}(1,35)=\right.$ $\left.59.646, p<.01 ; F_{2}(1,59)=56.560, p<.01\right]$ and preview $\left[F_{1}(2,70)=3.253, p=.039 ; F_{2}(2,118)=3.358, p=.035\right]$ yielded significant main effects. Under restricted skipping criteria, short target words were skipped $8 \%$ of the time and long target words were skipped $1 \%$ of the time. Target words were skipped on $6 \%$ of full-preview trials and $4 \%$ of both orthographically legal preview trials and orthographically illegal preview trials. Of trials initially recorded as first-pass skips, reclassification affected $35 \%$ of the cases, or exactly 48 trials $(2.5 \%$ of all critical trials). No interactions of length and preview were significant, using either method of calculating skipping proportions (all $F \mathrm{~s}<1$ ).

\section{Discussion}

The purpose of the present study was to assess the effects of word length and orthographic characteristics by manipulating the letter information available to readers during parafoveal processing. The use of frequency-matched long-short word pairs with identical initial letter trigrams allowed for the examination of word length effects on eye movement behavior, independent of many other lexical and sublexical features. The use of the invisible boundary paradigm permitted strong manipulations of parafoveal information without excessive disruption to subsequent foveal processing. The results confirmed prior research demonstrating that word length and orthographic information in the parafovea influenced initial landing positions during reading. Word length affected the incoming saccade amplitudes, initial fixation landing positions, and numbers of first-pass fixations. The orthographic characteristics of the target word preview affected the initial fixation landing positions and all first-pass reading times. The findings also indicated that, across both long and short words, parafoveal preview manipulations exerted similar effects on saccade characteristics. If readers were utilizing different amounts of orthographic information during saccade targeting as a function of word length, interactions of length and preview would have been observed.

Despite attempts to control as many lexical and sublexical characteristics as possible, there were large differences in orthographic neighborhood size across target word length 
conditions. Furthermore, the patterns of orthographic neighborhood size differences across preview conditions were not identical for the short and long target word conditions. That being said, if orthographic neighborhood size had an appreciable influence on saccade-targeting processes, one would expect this pattern of differences in orthographic neighborhood size across conditions to have generated an interaction of target word length and parafoveal preview. The facts that long target words are more likely to receive multiple firstpass fixations and have relatively lower lexical frequencies could also have contributed to the observed main effects of word length on the proportion into the target word for initial fixations. Nevertheless, the influence of lexical frequency was eliminated as much as possible by the selection of frequency-matched long-short target word pairs. It is also important to reiterate that some portion of the differences in the landing position distributions between length conditions can be attributed to the differential impacts of random and systematic oculomotor error. Systematic error, related to saccade launch site, and random error, related to eye movement targeting, necessarily have bigger impacts on shorter than on longer words. The spatial components of oculomotor error are more likely to place fixations in less-thanoptimal viewing locations for short than for long words, since short words take up less space. Simply put, no type of human motor plan is executed perfectly all of the time. In spite of the constant constraints imposed by oculomotor error, initial landing positions into both long and short words reflect precise targeting processes that are sensitive to information with both low (word length information) and high (letter identity information) spatial frequency that is retrieved during parafoveal processing.

When controlling for the influence of saccade launch site, which is a relevant predictive factor for initial landing positions, the critical results were the same. The linear mixedeffects regression model, which included the launch site of the critical saccade as a predictor, yielded a pattern of results identical to the one from a conventional ANOVA. Post hoc analyses revealed significant differences in landing positions for full-preview and orthographically illegal preview conditions, with average landing positions for orthographically legal previews falling between the two other conditions. This pattern of results was the same for the singlefixation restricted analyses and for landing position analyses on all first fixations. As is shown in Tables 1 and 2, letter strings comprising actual words are more frequently encountered in their respective positions than letter strings comprising orthographically legal nonwords. Likewise, the letter strings forming legal nonwords are more frequent than letter strings comprising illegal nonwords. Because the study included categorical manipulations of orthographic legality and lexical status, as well as a graded manipulation of orthographic familiarity and regularity (which subsume legality and are highly influenced by lexical status), the landing position results could reflect a combination of all three manipulations. The graded effect of orthographic regularity and familiarity on landing positions is quite similar to the effects Radach et al. (2004) reported. One possible explanation for the stepwise orthographic effect is a cumulative effect of orthographic processing difficulty in general, encompassing orthographic legality, regularity, and familiarity. However, it is not necessary to assume that all aspects of orthography are assessed simultaneously. Specifically, there is the possibility that orthographic legality, regularity, and familiarity are assessed independently or sequentially during parafoveal preprocessing. In such a system, letter sequence legality and familiarity would codetermine orthographic processing difficulty in conjunction with positionspecific letter sequence frequency. For example, the bigram $l t$ is frequently encountered at the end but not at the beginning of a word. Although this bigram is relatively frequent in English, it is neither familiar nor regular at the beginning of a word. Orthographic processing difficulty may result from letter sequences that are either illegal or infrequently encountered at a particular position. This kind of account would offer possible explanatory power for research demonstrating the flexibility of letter encoding during reading and nonreading tasks (Johnson \& Dunne, 2012; Johnson et al., 2007).

It is also possible that the legality of letter sequences is assessed in the initial stages of orthographic processing before regularity and familiarity exert an influence. If this is the case, an orthographically illegal initial letter sequence would elicit a larger deviation from the PVL, because its influence on saccade targeting is exerted earlier than that of orthographic regularity in general or lexical status. If orthographic-to-phonological activation routinely occurs during parafoveal letter processing, the observed landing position effects could also reflect an influence of phonological processing on eye movement programming. Indeed the results make it entirely possible that when a letter sequence encountered in the parafovea fails to activate an existing phonological representation, saccade-targeting strategies are affected. This effect could lead to readers either targeting saccades directly toward regions of phonological processing difficulty or generally adopting a more conservative saccade-targeting procedure ultimately resulting in shorter saccades. The increased likelihood that the initial letters of a legal nonword would activate existing phonological representations relative to an illegal nonword may have contributed to the observed landing position effects. Moreover, the increased likelihood of speeded phonological activation when processing actual words rather than pronounceable nonwords might also have contributed to the observed landing position results.

The landing position distributions across preview conditions suggest that the saccade programming system is 
capable of flexible fixation targeting. These findings fit well with a claim that the eye movement targeting system is capable of utilizing multiple sources of information at two levels of visual perception. One source of information, word length, is virtually always used and only requires the extraction of relatively low-spatial-frequency information. A second possible source, orthography, requires the extraction of high-spatial-frequency information, which may not always occur in time to influence saccade programming. Due to visual-acuity limitations, length information about a parafoveal target can be acquired from farther away than letter information. If parafoveal information is acquired and processed as soon as possible, length should affect the targeting system before orthography. The use of these two sources of information by the eye movement targeting system may also lead to initial landing positions reflecting two "centers of gravity," demonstrating both an imperative to process words from an optimal position (i.e., the PVL) and the inclination to target sites of detected processing difficulty within upcoming words. The eye movement control system would use the default center of gravity at the center of a saccade target and an additional center of gravity at a site of detectable orthographic processing difficulty. For short and long words, this would most likely result in a leftward shift to the peak of an initial fixation landing site distribution. The examination of a larger data set with greater statistical power could potentially demonstrate a bimodal initial fixation landing site distribution with one peak over the PVL and a second over regions of orthographic processing difficulty. Presumably, such a distribution could be demonstrated more clearly with longer words, since the spatial distance between the site of processing difficulty and the PVL would be large enough to differentiate various saccade target regions. Importantly, explanations positing an influence of general orthographic processing difficulty, distinct processing of orthography and phonology across several dimensions, a "center-of-gravity" modulatory effect, or any combination of the three are by no means mutually exclusive.

The first-pass reading times revealed a pattern similar to the landing position results with regard to orthographic feature effects. However, post hoc analyses showed that the first-pass reading measures differed significantly for identical parafoveal previews as compared to orthographically legal and illegal nonword preview conditions. Target words with identical previews received significantly shorter viewing times than did target words with orthographically legal or illegal previews. For the nonword previews, there was not a statistically significant effect of orthographic legality. The size of the preview benefit for full previews relative to orthographically legal and illegal nonwords suggests that a mismatch between the orthographic information extracted in the fovea and parafovea leads to processing difficulty. And while the lexical status of preview strings seems not to exert an effect on initial landing positions, it does affect first-pass reading times.

The results of the analyses on the pretarget adjective are surprising when considering the history of orthographic parafoveal-on-foveal effects observed in previous experimental research (White, 2008; White \& Liversedge, 2004). In a reading study similar to the present experiment, White and Liversedge (2004) failed to observe parafoveal-onfoveal effects on reading times for words preceding targets that varied in orthographic legality, pronounceability, familiarity, and informativeness. In their study, pretarget words were controlled using constraints similar to those in the present study; thus, the disparate findings could be indicative of intrinsic differences between the experimental materials or of the fragile nature of parafoveal-on-foveal effects in general. White (2008) observed that fixations immediately prior to entering critical words were slightly longer when the critical word contained less as compared to more familiar orthography. In the present study, single-fixation durations on the word preceding the target decreased numerically as orthographic processing difficulty in the parafovea increased across both length conditions. The differences between these patterns of results could be caused by the strength of the orthographic manipulation in the present study. In White's study, there was no parafoveal preview manipulation; the experimental conditions were achieved by independently manipulating the lexical frequency and orthographic familiarity of the critical words. Orthographic characteristics, in particular, were manipulated by selecting words with high or low orthographic familiarity; nonwords were never presented to subjects. Conversely, in the present study, orthographic characteristics were altered during parafoveal preview in order to manipulate potential processing difficulty. This substitution of target words with nonwords produced the strongest possible manipulation of orthographic characteristics and the largest differences between target word parafoveal preview conditions within identical sentence frames. This procedural difference makes any comparison of the two studies difficult without making strong assumptions about the influence of the lexical status of a letter string on parafoveal processing. The apparent parafoveal-on-foveal effect fits well into "popout" accounts of orthography's influence on saccade targeting (Beauvillain et al., 1996; Findlay \& Walker, 1999; Hyönä, 1995). Single-fixation durations on the pretarget adjective decreased as the orthographic regularity of the initial letters of the upcoming preview string decreased. Under the assumptions of a pop-out account, this effect was observed because orthographic abnormalities are easily detectable. As parafoveal abnormalities become more pronounced, the likelihood of attracting attention increases, and may even prompt the eye movement control system to terminate current fixations prematurely. Although eye 
movements and visual attention are not necessarily coupled, a shift in attention will often be followed by an eye movement. Prior studies have shown that short words are skipped more often than long words during first-pass reading (Brysbaert \& Vitu, 1998; Rayner \& McConkie, 1976; Rayner et al., 1996; Rayner et al., 2011). Previous research has also documented a tendency for fixation durations to be inflated before first-pass word skips (Kliegl \& Engbert, 2005; Pollatsek, Rayner, \& Balota, 1986; Rayner, Ashby, Pollatsek, $\&$ Reichle, 2004). If the difference in single-fixation durations preceding initial target fixation was driven by an increased tendency to program first-pass skips of the target word under full-preview conditions, one might expect an interaction in single fixations, whereby fixation times were inflated for short but not for long target words in full-preview conditions. Our results showed skipping proportions in the expected pattern; nevertheless, there were no statistically significant interactions of length and preview. For both length conditions, the skipping proportions across preview conditions went in the direction one would expect if the parafoveal-on-foveal effect was driven by first-pass skipping. Considering these data, increased durations associated with executing first-pass target word skips remain the most well defined and supportable explanation. Given the controversial nature of parafoveal-onfoveal effects, further investigation of orthographic and lexical processing in the parafovea is needed in order to illuminate the influence of upcoming letters on processing in the fovea.

In summary, the present data demonstrate that extraction of orthographic information from parafoveal saccade targets influences targeting processes. This orthographic influence on landing position is unaffected by the length of a saccade target. These results also provide evidence that orthographically inaccurate parafoveal previews disrupt subsequent processing once the saccade target is fixated. The present study supports previous studies suggesting that the eye movement targeting system has both the sensitivity to detect regions of orthographic processing difficulty and the necessary flexibility to aim saccades into those specific regions. Furthermore, these results demonstrate that this sensitivity is not modulated by the length of a saccade target.

Author note This research was supported by Grant HD26765 from the National Institute of Child Health and Human Development and was conducted when the first author held a predoctoral fellowship on Grant T32DC00041 from the Center for Research in Language. The authors thank Bernhard Angele, Victor Ferreira, Leslie Carver, Karen Dobkins, Liz Schotter, Barbara Juhasz, Simon P. Liversedge, and an anonymous reviewer for their helpful comments on earlier versions.

\section{References}

Angele, B., \& Rayner, K. (2011). Parafoveal processing of word $n+2$ during reading: Do the preceding words matter? Journal of
Experimental Psychology: Human Perception and Performance, 37, 1210-1220.

Angele, B., Slattery, T. J., Yang, J., Kliegl, R., \& Rayner, K. (2008). Parafoveal processing in reading: Manipulating $n+1$ and $n+2$ previews simultaneously. Visual Cognition, 16, 697-707.

Baayen, R. H., Piepenbrock, R., \& Gulikers, L. (1995). The CELEX lexical database (Release 2, CD-ROM). Philadelphia: Linguistic Data Consortium, University of Pennsylvania.

Balota, D. A., Yap, M. J., Cortese, M. J., Hutchison, K. A., Kessler, B., Loftis, B., . . Treiman, R. (2007). The English Lexicon Project. Behavior Research Methods, 39, 445-459. doi:10.3758/ BF03193014

Beauvillain, C., Doré, K., \& Baudouin, V. (1996). The "centre of gravity" of words: Evidence for an effect of word-initial letters. Vision Research, 36, 589-603.

Brysbaert, M., \& Vitu, F. (1998). Word skipping: Implications for theories of eye movement control in reading. In G. Underwood (Ed.), Eye guidance in reading and scene perception. 125-148

Davis, C. J. (2005). N-Watch: A program for deriving neighborhood size and other psycholinguistic statistics. Behavior Research Methods, 37, 65-70. doi:10.3758/BF03206399

Drieghe, D., Rayner, K., \& Pollatsek, A. (2005). Eye movements and word skipping during reading revisited. Journal of Experimental Psychology: Human Perception and Performance, 31, 954-969. doi:10.1037/0096-1523.31.5.954

Everatt, J., \& Underwood, G. (1992). Parafoveal guidance and priming effects during reading: A special case of the mind being ahead of the eyes. Consciousness and Cognition, 1, 186-197.

Findlay, J. M., \& Walker, R. (1999). A model of saccade generation based on parallel processing and competitive inhibition. Behavioural and Brain Sciences, 22, 661-721.

Hyönä, J. (1995). Do irregular letter combinations attract readers' attention? Evidence from fixation locations in words. Journal of Experimental Psychology: Human Perception and Performance, 21, 68-81. doi:10.1037/0096-1523.21.1.68

Hyönä, J., Niemi, P., \& Underwood, G. (1989). Reading long words embedded in sentences: Informativeness of word parts affects eye movements. Journal of Experimental Psychology: Human Perception and Performance, 15, 142-152.

Hyönä, J., \& Pollatsek, A. (1998). Reading Finnish compound words: Eye fixations are affected by component morphemes. Journal of Experimental Psychology: Human Perception and Performance, 24, 1612-1627. doi:10.1037/0096-1523.24.6.1612

Hyönä, J., \& Pollatsek, A. (2000). Processing of Finnish compound words in reading. In A. Kennedy, R. Radach, D. Heller, \& J. Pynte (Eds.), Reading as a perceptual process (pp. 65-87). Amsterdam: Elsevier.

Inhoff, A. W., Radach, R., Eiter, B., \& Juhasz, B. (2003). Distinct subsystems for the parafoveal processing of spatial and linguistic information during eye fixations in reading. Quarterly Journal of Experimental Psychology, 56A, 803-827.

Inhoff, A. W., Starr, M., Liu, W., \& Wang, J. (1998). Eye-movementcontingent display changes are not compromised by flicker and phosphor persistence. Psychonomic Bulletin \& Review, 5, 101106.

Johnson, R. L., \& Dunne, M. D. (2012). Parafoveal processing of transposed-letter words and nonwords: Evidence against parafoveal lexical activation. Journal of Experimental Psychology: Human Perception and Performance, 38, 191-212. doi:10.1037/ a0025983

Johnson, R. L., Perea, M., \& Rayner, K. (2007). Transposed-letter effects in reading: Evidence from eye movements and parafoveal preview. Journal of Experimental Psychology: Human Perception and Performance, 33, 209-229. doi:10.1037/0096-1523.33.1.209

Juhasz, B. J., White, S. J., Liversedge, S. P., \& Rayner, K. (2008). Eye movements and the use of parafoveal word length information in 
reading. Journal of Experimental Psychology: Human Perception and Performance, 34, 1560-1579. doi:10.1037/a0012319

Kliegl, R., \& Engbert, R. (2005). Fixation durations before word skipping in reading. Psychonomic Bulletin \& Review, 12, 132 138.

Lund, K., \& Burgess, C. (1996). Producing high-dimensional semantic spaces from lexical co-occurrence. Behavior Research Methods, Instruments, \& Computers, 28, 203-208. doi:10.3758/ BF03204766

McConkie, G. W., Kerr, P. W., Reddix, M. D., \& Zola, D. (1988). Eye movement control during reading: I. The location of initial eye fixations on words. Vision Research, 28, 1107-1118.

McDonald, S. A. (2006). Effects of number-of-letters on eye movements during reading are independent from effects of spatial word length. Visual Cognition, 13, 89-98. doi:10.1080/135062 80500143367

Morris, R. K., Rayner, K., \& Pollatsek, A. (1990). Eye movement guidance in reading: The role of parafoveal letter and space information. Journal of Experimental Psychology: Human Perception and Performance, 16, 268-281. doi:10.1037/00961523.16.2.268

Perea, M., \& Pollatsek, A. (1998). The effects of neighborhood frequency in reading and lexical decision. Journal of Experimental Psychology: Human Perception and Performance, 24, 767-777.

Pollatsek, A., Perea, M., \& Binder, K. (1999). The effects of neighborhood size in reading and lexical decision. Journal of Experimental Psychology: Human Perception and Performance, 25, $1142-1158$

Pollatsek, A., \& Rayner, K. (1982). Eye movement control in reading: The role of word boundaries. Journal of Experimental Psychology: Human Perception and Performance, 8, 817-833.

Pollatsek, A., Rayner, K., \& Balota, D. A. (1986). Inferences about eye movement control from the perceptual span in reading. Perception \& Psychophysics, 40, 123-130. doi:10.3758/BF03208192

Radach, R., Inhoff, A., \& Heller, D. (2004). Orthographic regularity gradually modulates saccade amplitudes in reading. European Journal of Cognitive Psychology, 16, 27-51.

Rayner, K. (1975). The perceptual span and peripheral cues in reading. Cognitive Psychology, 7, 65-81.

Rayner, K. (1979). Eye guidance in reading: Fixation locations within words. Perception, 8, 21-30.

Rayner, K. (1998). Eye movements in reading and information processing: 20 years of research. Psychological Bulletin, 124, 372 422. doi:10.1037/0033-2909.124.3.372

Rayner, K. (2009). The Thirty Fifth Sir Frederick Bartlett Lecture: Eye movements and attention in reading, scene perception, and visual search. Quarterly Journal of Experimental Psychology, 62, 14571506.

Rayner, K., Ashby, J., Pollatsek, A., \& Reichle, E. D. (2004). The effects of frequency and predictability on eye fixations sin reading: Implications for the E-Z Reader model. Journal of Experimental Psychology: Human Perception and Performance, 30, $720-732$.

Rayner, K., Binder, K. S., Ashby, J., \& Pollatsek, A. (2001). Eye movement control in reading: Word predictability has little influence on initial landing positions in words. Vision Research, 41, 943-954.
Rayner, K., Fischer, M. H., \& Pollatsek, A. (1998). Unspaced text interferes with both word identification and eye movement control. Vision Research, 38, 1129-1144.

Rayner, K., \& McConkie, G. W. (1976). What guides a reader's eye movements? Vision Research, 16, 829-837.

Rayner, K., \& Morris, R. K. (1992). Eye movement control in reading: Evidence against semantic preprocessing. Journal of Experimental Psychology: Human Perception and Performance, 18, 163172.

Rayner, K., \& Pollatsek, A. (1981). Eye movement control during reading: Evidence for direct control. Quarterly Journal of Experimental Psychology, 33A, 351-373.

Rayner, K., \& Pollatsek, A. (1989). The psychology of reading. Englewood Cliffs: Prentice Hall.

Rayner, K., Sereno, S. C., \& Raney, G. E. (1996). Eye movement control in reading: A comparison of two types of models. Journal of Experimental Psychology: Human Perception and Performance, 22, 1188-1200.

Rayner, K., Slattery, T. J., Drieghe, D., \& Liversedge, S. P. (2011). Eye movements and word skipping during reading: Effects of word length and predictability. Journal of Experimental Psychology: Human Perception and Performance, 37, 514-528.

Rayner, K., Well, A. D., Pollatsek, A., \& Bertera, J. H. (1982). The availability of useful information to the right of fixation in reading. Perception \& Psychophysics, 31, 537-550. doi:10.3758/ BF03204186

Schotter, E. R., Angele, B., \& Rayner, K. (2012). Parafoveal processing in reading. Attention, Perception, \& Psychophysics, 74, 5-35. doi:10.3758/s13414-011-0219-2

Spragins, A. B., Lefton, L. A., \& Fisher, D. F. (1976). Eye movements while reading spatially transformed text: A developmental study. Memory \& Cognition, 4, 36-42.

Underwood, G., Clews, S., \& Everatt, J. (1990). How do readers know where to look next? Local information distributions influence eye fixations. Quarterly Journal of Experimental Psychology, 42A, $39-65$.

Underwood, N. R., \& McConkie, G. W. (1985). Perceptual span for letter distinctions during reading. Reading Research Quarterly, $20,153-162$.

White, S. J. (2008). Eye movement control during reading: Effects of word frequency and orthographic familiarity. Journal of Experimental Psychology: Human Perception and Performance, 34, 205-223.

White, S. J., Johnson, R. L., Liversedge, S. P., \& Rayner, K. (2008). Eye movements when reading transposed text: The importance of word-beginning letters. Journal of Experimental Psychology: Human Perception and Performance, 34, 1261-1276. doi:10.1037/0096-1523.34.5.1261

White, S. J., \& Liversedge, S. P. (2004). Orthographic familiarity influences initial eye fixation positions in reading. European Journal of Cognitive Psychology, 16, 52-78.

White, S. J., \& Liversedge, S. P. (2006a). Foveal processing difficulty does not modulate non-foveal orthographic influences on fixation positions. Vision Research, 46, 426-437.

White, S. J., \& Liversedge, S. P. (2006b). Linguistic and non-linguistic influences on the eyes' landing positions during reading. Quarterly Journal of Experimental Psychology, 59, 760-782. 